\title{
Hydrophobicity in clearwing butterflies and moths: impact of scale micro and nanostructure, and trade-off with optical transparency
}

Doris Gomez ${ }^{\star}$, Jonathan Pairraire ${ }^{2}$, Charline Pinna ${ }^{3}$, Monica Arias $^{1}$, Céline Houssin ${ }^{3}$, Jérôme Barbut ${ }^{3}$, Serge Berthier ${ }^{2}$, Christine Andraud ${ }^{4}$, Thierry Ondarçuhu ${ }^{5}$, Marianne Elias ${ }^{3}$

${ }^{1}$ CEFE, CNRS, Univ Montpellier, EPHE, IRD, Montpellier, France, 34090

2 INSP, Sorbonne University, CNRS, Paris, France, 75005

${ }^{3}$ Institut de Systématique, Evolution, Biodiversité (ISYEB), CNRS, Muséum national d'Histoire naturelle, Sorbonne Université, EPHE, Université des Antilles, France, 75005

${ }^{4}$ CRC, MNHN, Paris, France, 75005

5 IMFT, Univ. Toulouse, CNRS, Toulouse, France, 31400

*Doris Gomez

Email: doris.gomez@cefe.cnrs.fr

Author Contributions: DG, ME, CA and SB designed the study, DG, ME, CA, JP, and CP defined the protocol, JB, ME, and DG selected the specimens, DG, JP and CH took the structure, CA, and optical measurements, DG, TO, and MA analyzed the data, and all authors contributed to manuscript writing.

Competing Interest Statement: No competing interests.

Classification: major: Physics and Evolution; minor: Ecology

Keywords: Lepidoptera; transparency; trade-off; multiscale roughness; hydrophobicity

This PDF file includes:

Main Text

Figures 1 to 8 


\section{Abstract $(245<=250$ words, up to three non-numerical references)}

In opaque butterflies and moths, scales ensure vital functions like camouflage, thermoregulation, and hydrophobicity. Wing transparency in some species - achieved via modified or absent scales - raises the question of whether hydrophobicity can be maintained and of it dependence on scale microstructural (scale presence, morphology, insertion angle, and coloration) and nanostructural (ridge spacing and width) features. To address these questions, we assessed hydrophobicity in 23 clearwing species differing in scale micro and nanofeatures by measuring static contact angle $(C A)$ of water droplets in the opaque and transparent patches of the same individuals at different stages of evaporation. We related these measures to wing structures (macro, micro, and nano) and compared them to predictions from Cassie-Baxter and Wenzel models. We found that overall, transparency is costly for hydrophobicity and this cost depends on scale microstructural features: transparent patches are less hydrophobic and lose more hydrophobicity with water evaporation than opaque patches. This loss is attenuated for higher scale densities, coloured scales (for erect scales), and when combining two types of scales (piliform and lamellar). Nude membranes show lowest hydrophobicity. Best models are Cassie-Baxter models that include scale microstructures for erect scales, and scale micro and nanostructures for flat scales. All findings are consistent with the physics of hydrophobicity, especially on multiscale roughness. Finally, wing hydrophobicity negatively relates to optical transparency. Moreover, tropical species have more hydrophobic transparent patches but similarly hydrophobic opaque patches compared to temperate species. Overall, diverse microstructures are likely functional compromises between multiple requirements.

\section{Significance Statement $(=119,>50$ and $<=120$, no references)}

Water repellency is vital for terrestrial organisms. Yet, how microstructural diversity may impact hydrophobicity is unknown. Bridging the gap between biology and physics, we exploit the microstructural diversity found in clearwing butterflies and moths to assess its impact on hydrophobicity, and its ecological relevance. Within a physical framework, we bring experimental and modelling evidence for a major role of microstructures (scale morphology, insertion angle, coloration) and multiscale roughness in determining wing hydrophobicity, with a role of nanostructures restricted to flat scales and nude membrane. For the first time, we evidence some costs for transparency, and a trade-off between optics and hydrophobicity. Beyond novel biological results, this study gives new sources of bioinspiration for applied research on transparent materials in physics. 


\section{Main Text $(<8000$ words)}

36

\section{Introduction}

Hydrophobicity is essential for terrestrial organisms. As predicted by physics (1) and illustrated in plants (2), a key parameter for hydrophobicity is surface texture or roughness. A water droplet sitting on a textured hydrophobic surface can exhibit two different wetting states. First, in the Cassie-Baxter state (Figure 1, series a), the water droplet sits on top of the texture, with trapped air underneath and cavities filled with air (composite state: under the drop, water can be in contact with solid or air), and hydrophobicity is at a maximum. If this state is thermodynamically unstable, the water droplet may undergo the so-called Cassie-Baxter to Wenzel transition, in which water penetrates the air-filled cavities by capillarity. In the Wenzel wetting state (Figure 1 series $b$ ), the water droplet fully fills all the cavities of the textured surface and adheres to the surface (non-composite state: under the drop, water is in contact with solid only and no longer with air), decreasing the surface energy; hydrophobicity is then lost (3). Compared to the Wenzel state, the Cassie-Baxter state is of high biological interest as it offers an incomplete water-surface contact and a weak water adhesion. Maintaining a stable Cassie-Baxter state is crucial to maintain high hydrophobicity under harsh environmental conditions, rainfall for instance. Roughness at nanoscale - the parameter most studied to date in animals and plants - increases hydrophobicity, as shown in cicadids and dragonflies (4-6). Yet, multiscale roughness -at nano and micro scale - is even more efficient: it increases hydrophobicity and its thermodynamic stability (in modelling studies 7, 8, illustrated in the Lotus (so-called 'Lotus effect') and other plants in 9) and reduces water adhesion (7). Increasing thermodynamic stability allows maintaining hydrophobicity with water droplets of various sizes (dew, fog, rain) and increases anti-fogging properties, i. e. the resistance to tiny water droplets condensing on the surface. While the role of nanostructures in hydrophobicity has been extensively documented (e.g. 10, 11), the role of microstructures shape in determining hydrophobic properties has been limited to simple geometries (cones in 12, cylinders in 13,14) and remains poorly investigated from an empirical perspective. The only existing empirical studies with such an approach either focus on one type of micro-architecture $(6,15)$ thereby excluding microstructural influence, or they describe variation in hydrophobicity between various micro-architectures but without invoking explanations (16).

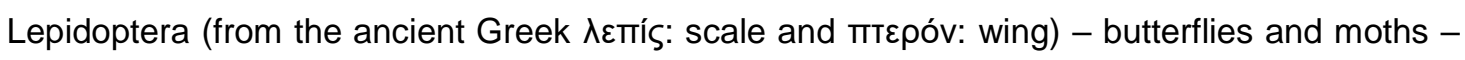
offer an outstanding group to investigate this question. They are characterized by wings entirely covered with flat and coloured lamellar scales (17). Through their pigmentation and structure, scales are involved in multiple functions such as antipredator defences (e. g. camouflage, deflection, mimicry in 18, 19), communication (20), thermoregulation (17, 21-23), or flight enhancement $(24,25)$. They also confer superhydrophobic properties to the wing, resulting in water repellency and self-cleaning $(26,27)$. Superhydrophobicity sensu lato is defined by water droplets making high contact angles $\left(>150^{\circ}\right)$ with a surface. Self-cleaning - superhydrophobicity stricto sensu (definition not taken here) - adds to this condition a weak water adhesion, estimated by a minimal tilt from the horizontal plane needed for water droplets to roll-off (roll-off angle of a few degrees) or a minimal hysteresis (difference between advancing 
and receding contact angles). Superhydrophobicity is thus a sine qua non condition for water repellency and self-cleaning. Opaque butterflies and moths typically have self-cleaning wings, as attested by small roll-off angles $(15,28)$. Scarce relevant studies suggest that wing hydrophobicity may depend on wing microstructure (presence and type of scale in 26, scale type and insertion angle in 29), and on wing macrostructure: species with longer wings (4), or larger ratio of wing area to body mass (26) show higher hydrophobicity and wing shape was invoked to explain natural variations in hydrophobicity (4).

While the vast majority of Lepidoptera species has opaque wings, some species from various lineages show transparent or translucent wings (30), which reduces their detectability from visuallyhunting predators (31-33). Transparency shows a broad microstructural diversity (i. e. scale diversity, see examples in Figure 2), the membrane being nude or covered with scales varying in type (piliform, i. e., hair-like, and/or lamellar), insertion on the membrane (flat or erect), and colouration (coloured or transparent) (30). All combinations of scale type, insertion, and colouration (i. e., structural strategies 30) can be found in nature (Figure 2), and they differ in their efficiency at transmitting light : the nude membrane are most efficient while flat coloured scales (lamellar alone or in combination with piliform) are least efficient (30). Microstructures are complemented by nanostructures: longitudinal ridges on scales, and nanostructures on the wing membrane, of various shape and density. Membrane nanostructures reduce reflection levels and increase light transmission (34-37).

Because transparency often entails profound modifications of scale dimensions and density (30), we can suppose that transparency may be potentially costly for hydrophobicity, for both water repellency and self-cleaning. These functions are vital for butterflies and moths: water repellency is crucial for flight and for preventing wings from sticking together, especially in tropical rainforest species with daily rain and high humidity. Likewise, self-cleaning helps removing dust contamination that impairs flight (26). Among the lepidopteran species investigated so far for hydrophobicity $(15,26,27,29,38)$, only three clearwing butterfly species have been included: Parantica sita (with lamellar titled scales) and Parnassius glacialis (with flat lamellar scales), with high or moderate hydrophobicity respectively (15, 29), and Greta oto (with piliform scales) with one of the lowest hydrophobicity values found in butterflies (27). Scarce data suggest that a greater reduction in scale dimensions or coverage on the wing membrane may entail higher costs in terms of hydrophobicity. However, large-scale comparative studies are currently lacking.

To fill that gap in our knowledge, here we explore to what extent anti-wetting ability is influenced by micro and nanostructure in species that largely differ in their wing microstructure, by selecting a subset of 23 species (Figure 3 ) from a broad study of 123 clearwing Lepidoptera species (30). In these species, we explored the links between structure, hydrophobicity and optics while controlling for phylogenetic relatedness between species. First, we explored the relationships between hydrophobicity and wing structure, at macro-, micro- and nano-structural level: (i) we measured the contact angle (CA) made by water droplets of various sizes on the wing (ii) We then quantified wing macro-, micro-, and nanostructures, and related them to hydrophobicity. (iii) Using a modelling perspective, we ran various Cassie-Baxter and Wenzel models differing by their assumptions and compared the predicted to the observed CA values to assess the relative importance of microstructure and nanostructures in determining the observed hydrophobicity. Second, to identify the selective pressures acting on 
hydrophobicity, we tested whether hydrophobicity and light transmission showed potential trade-off or synergy. If microstructures play a dominant role in conferring hydrophobicity, species most efficient at transmitting light - which lack scales or have highly modified scales in low coverage on the wing surface - are expected be less efficient at repelling water. Third, to identify whether hydrophobicity is influenced by environmental conditions, we tested the links between habitat latitude and hydrophobicity: if repelling water is more important in the tropics where rain and humidity are inescapable, tropical species are expected to show higher hydrophobicity than temperate species.

\section{Results \& Discussion}

124

We measured the contact angle of water droplets and wing surface in the transparent and opaque zones of the forewing of three museum specimens per species, and we monitored contact angle at three times, as water evaporated and droplet size decreased (Figures 2 and S1). We here considered hydrophobicity as a proxy for self-cleaning ability. Indeed, although we could not quantify water droplet roll-off angles precisely with our set-up, we observed that water droplets rolled off extremely fast from the wings when not perfectly horizontal (DG, ME, JP, and $\mathrm{CH}$ pers. obs), which made our measurements particularly time-consuming. This suggested small roll-off angles and weak water adhesion. Small roll-off angles are commonly found in opaque butterflies $(15,28)$ and in Parantica sita and Parnassius glacialis, the two clearwing butterfly species studied to date (29).

We observed a general decrease in hydrophobicity with water evaporation in the opaque zone and in the transparent zone (Figure 4A). Such a decrease is commonly observed in hydrophobic humanmade surfaces $(14,39,40)$ and in natural surfaces, as in the transparent-winged damselfly Ischnura heterosticta (3). It is interpreted as a loss of self-cleaning ability when contact angle values get below the hydrophilicity threshold (3). Beyond this general trend, two results showed that transparency entails potential costs in terms of water repellency and self-cleaning ability. First, the transparent zone showed lower hydrophobicity than the opaque zone of the same wing, whatever the size of the water droplet considered (Figure 4A, zone effect in Table S1, see Figure S2 for distribution of hydrophobicity levels). Second, we observed a stronger decrease in hydrophobicity with water evaporation in the transparent than in the opaque zone of the same wing (time $x$ zone effect in Table S1, Figure 4A).

\section{Variation in hydrophobicity and relation to wing macrostructure}

Relationships between hydrophobicity and wing macrostructure were surprising, probably due to the taxonomic level investigated. Contrary to Byun et al.'s (4) finding that contact angle positively correlated to wing length (24 species, 10 insect orders), CA as variable to explain, wing length as factor, estimate $=1.12 \pm 0.38, \mathrm{t}=2.93, \mathrm{p}=0.008$ ), we found no relationship between hydrophobicity and wing length (Table S1). Wing shape was much more diverse in Byun et al.'s dataset (LWratio: $\min =1.2$, mean=3.6, $\max =9.3$ ) than in ours ( 23 species, 1 insect order), LWratio: $\min =1.8$, mean=2.2, $\max =3.2$ ) When restricting their dataset to our maximal value for LWratio, the effect of wing length was no longer 
significant in Byun's dataset $(n=13$, wing length estimate $=-23.48 \pm 13.42, t=-1.74, p=0.13)$ and the null model performed best.

Contrary to Wagner et al.'s (26) finding that hydrophobicity positively correlated to the ratio of wing area to body mass (38 species (14 insect orders, CA as variable to explain, ratio of wing area to body mass as factor, estimate $=0.75 \pm 0.25, \mathrm{t}=2.95, \mathrm{p}=0.007$ ), we found an important negative correlation (Table S1, WingArea / BodyVolume effect, only important when correcting for phylogeny, Figure 4C). When restricting Wagner et al's dataset to insects without elytra but with a microstructured membrane (Odonata, Ephemeroptera, Lepidoptera, some Planipennia), Wagner et al.'s positive relationship was no longer important ( $n=21$ species ( 4 insect orders), wing area to body mass estimate $=0.01 \pm 0.08, t=-$ $0.14, p=0.89$ ) and the null model performed best.

Comparing our results to previous findings show that relationships with macrostructure seems dependent on the taxonomic scale and homogeneity in structure. Contrary to our expectation, species with more elongated wings (higher FW ratio) had a lower hydrophobicity (Figure $4 \mathrm{C}$ ). Water droplets exert higher moment force when further away from the butterfly body and should select for higher hydrophobicity; yet, more elongated wings may already show higher elasticity that may already ensure rapid droplet roll-off through movement, and offset the need for a higher hydrophobicity. Finally, we found that species with more elongated wings or with shorter wings exhibited a greater loss of hydrophobicity with evaporation.

\section{Variation in hydrophobicity and relation to wing microstructure}

We performed classic mixed models and Bayesian models, the latter controlling for species phylogenetic relatedness, to test to what extent hydrophobicity depends on wing microstructure - namely scale presence, type (piliform and/or lamellar), insertion (erect or flat on the wing membrane), coloration (transparent or coloured), and density in both the transparent and the opaque zones - and wing scale nanostructures - namely the width and spacing of longitudinal ridges that were present on all scales. Comparing models controlling or not for phylogeny helped us purge our results from spurious correlations arising from phylogenetic ancestry.

The influence of wing microstructure on hydrophobicity was pervasive in our results (Figure 5): (i) we found a higher interspecific variance in contact angle values in the transparent than in the opaque zone (Figure 4A, Fligner-Killeen tests with all times together $\chi^{2}=79.48, p<0.001$ or separated at T1: $\chi^{2}=49.57, \mathrm{p}<0.001 ; \mathrm{T} 2: \chi^{2}=29.24, \mathrm{p}<0.001 ; \mathrm{T} 3 \chi^{2}=26.47, \mathrm{p}<0.001$ ), maybe in relation to the higher interspecific microstructural diversity of the transparent zone. (ii) The nude membrane (N) yielded a lower hydrophobicity but a similar decrease in hydrophobicity compared to the structural strategies that involved scales (Table S2, scale presence effect in Table S3a, Figure 5). (iii) Combining two types of scales (piliform and lamellar) yielded comparable levels of hydrophobicity, but a lower decrease in hydrophobicity with evaporation than having only one type of scales only (Table S2, Scale Nb $(2>1) \mathrm{x}$ Time interaction in Table S3b, Figure 5). (iv) The decrease in hydrophobicity with evaporation was lower for erect coloured scales than for erect transparent scales (Table S2, Colour EC>ET x Time interaction in Table S3c, Figure 5). (v) The decrease in hydrophobicity with water evaporation was attenuated for denser scales (Table S2, time $x$ density interaction in Table S3c, Figure 4B). This attenuation by density 
was stronger in the transparent zone than in the opaque zone (Table S2, time $x$ density $x$ zone interaction in Table S3a, S3b, S3d, Figure 4B), and for erect scales (Table S2, time $x$ density interaction in Table S3c). (vi) For flat lamellar scales, the decrease in hydrophobicity with evaporation was attenuated when scales were arranged in a higher number of layers, be they in the transparent or in the opaque zone (Table S2, time x nb layers interaction in Table S3d). (vii) Finally, scale nanostructures did not explain an important part of the variation in hydrophobicity (scale ridge ratio never retained in the best models). Because erect geometries (involving piliform and/or lamellar scales: PLE, PE, LE) appear to behave differently in reaction to water than flat geometries, we analysed scale dimensions and spacing by performing classic mixed models and Bayesian analyses on the broad dataset of 123 species used by Gomez et al. (30) to get more representative trends. Compared to flat scales, erect scales were shortened when one type of scale was involved, especially in lamellar scales (Figure S3). When both present, piliform and lamellar scales were in similar densities, close in space, and piliform scales were 2.6 times longer than lamellar scales, creating a multi-hierarchical roughness at microscopic scales (see SI for supplementary results, Table S4, Figure S4).

For modelling, we first assigned water droplets at $\mathrm{T} 1$ to a Cassie-Baxter regime when droplets showed a high contact angle after evaporation $\left(C A \geq 120^{\circ}\right.$ at $\mathrm{T} 3$; results remained the same when shifting the threshold to $110^{\circ}$ ). We tested Cassie-Baxter models with microstructures alone (scale dimensions, insertion, and density) or with scale microstructures and nanostructures (ridge-ratio) to assess their relative importance in determining hydrophobicity observed at $\mathrm{T} 1$, selecting as best the model that minimized the difference between predictions and observations, for each structural strategy separately (SI, Figure S5 for model details, Figure $\mathrm{S} 6$ for best model selection). The best model included only microstructures for all erect strategies (with piliform and/or lamellar scales PE, LE, PLE, with a mean difference between observations and predictions of $19^{\circ}, 14^{\circ}$, and $7^{\circ}$ respectively) and for flat lamellar scales in low densities (not fully covering the wing membrane, with a mean difference between observations and predictions of $20^{\circ}$ and $22^{\circ}$ for the transparent and the opaque zone respectively). This is consistent with the fact that in these cases water is in contact with scale contour/edge which does not exhibit any nanostructures. For strategies involving erect piliform scales (PE, PLE), the model with bending piliform scales outperformed the model with fully erect piliform scales. For flat piliform scales (PF) and for flat lamellar scales (LF) in high densities (fully covering the membrane), the best model included microstructures and scale nanostructures (with a mean difference between observations and predictions of $12^{\circ}$ for $\mathrm{PF}, 20^{\circ}$ and $22^{\circ}$ for the transparent and the opaque zone respectively). In this case, the scale upper side is in contact with water and both types of structures come into play. In general, predictions were rather close to observations with no systematic bias towards under or over-estimation of $\Phi_{S}$, the fraction of the droplet contact area where water is in contact with the solid (Figure 6).

For the water droplets not categorized as in a Cassie-Baxter state, we tested whether a Wenzel model with microstructures only could predict the observed values at T1 (Figure S7). We could not test a Wenzel model with micro and nanostructures, as it required to precisely characterize scale nanogroove height and membrane nanostructures, which was not possible on museum specimens. Predictions of Wenzel models were far below observations (Figure S8). This is not surprising since the validity of Wenzel model - as currently formulated - is widely questioned in the literature (41 and 
references therein). This is especially true for our dataset given that most microstructures only weakly increase the roughness parameter $r$ (see materials and methods) while providing strong pinning sites for the contact line and thus significantly increasing the contact angle measured at T1.

Going back to the physical theory behind hydrophobicity, several studies have shown that a single-level structure does not necessarily guarantee a low water adhesion, even in the Cassie-Baxter state (see references in 7). Introducing higher levels of hierarchy increases the robustness of a surface hydrophobicity (8): it stabilizes the Cassie-Baxter state by dramatically decreasing the contact area fraction (ratio of contact area to the total surface area of the structure) and thus the adhesion force of water droplets, and by enlarging the energy difference between the Cassie-Baxter and the Wenzel states. Hierarchical structures can be frequently found in plants and in animals. For instance, in the water strider Gerris remigis, leg water resistance is due to the hierarchical structures of nano-grooved microsetae, which prevents striders from being drowned under heavy rainfall (42). This likely explains why, in our study, the combination of erect piliform scales and lamellar scales yields a lower loss of hydrophobicity with evaporation than piliform or lamellar scales alone. Such geometries have a 3-level roughness: (1) erect piliform scales bending over lamellar scales (piliform scales are 2.6 times longer than lamellar scales and first in contact with water), (2) erect lamellar scales tightly associated in space to piliform scales (similar density and close spacing), and (3) nanostructures on scales and on the wing membrane. Hydrophobicity likely results from the combination of the complex geometry of erect microstructures (which considerably reduces the proportion of the total surface in contact with water), and the gain in mechanical resistance (gain in elasticity and resistance against breakage) of piliform scales when bending against lamellar scales. The importance of elasticity of bending hair-like microstructures has been found in several studies. In the Lady's mantle plant (Alchemilla vulgaris), hairs are hydrophilic when measured individually, but they bend and coalesce into bundles when in contact with water droplets; their elasticity results in a repulsive interaction between the droplet and the plant surface, which maintains hydrophobicity (CA above $90^{\circ}$ ) (43). Likewise, in Nasutitermes termits, large bending hairs and small micrasters (micraster wavelength was around $11,7 \mu \mathrm{m}$ according to our measurements taken on Figure $3 \mathrm{C}$ from 41$)$ enable hydrophobicity $\left(\mathrm{CA}\right.$ above $\left.90^{\circ}\right)$ in both rain and mist conditions (44).

Increasing the density of microstructures does not significantly change hydrophobicity, but leads to a lower loss of hydrophobicity with water evaporation, i. e., to a higher resistance to tiny water droplets. This can be seen for all structural strategies (effect time $x$ density) and for flat scales organized in layers (effect time $\mathrm{x}$ number of layers). This is consistent with the fact that, during droplet evaporation, the Cassie-Baxter regime is more robust for large microstructure density. Not only scale architecture but also coloration can contribute to hydrophobicity. Erect scales show a lower loss of hydrophobicity when pigmented than when transparent. In the transparent zone, coloured scales exhibit colours ranging from pale yellow to brown and black. They are likely impregnated by melanin pigments, which are known to be involved - for some biochemical forms - in cuticle sclerotization (hardening) (45). Hence, the additional hardening conferred by pigments may increase their mechanical resistance to deformation and may contribute to maintaining hydrophobicity, even when evaporation occurs. 
We found that scale nanostructures did not contribute significantly to wing hydrophobicity for most structural strategies, except for flat lamellar scales fully covering the wing membrane and organized in layers, in the transparent or in the opaque zone, and for flat piliform scales in the transparent zone (Figure S6). Our results bring novel evidence for a major role of microstructures in explaining large variations in hydrophobicity when diverse microstructures are considered. The rare existing studies on the subject suggest a synergetic effect of scale nanostructures and microstructures on enhancing surface hydrophobicity (experiments on one type of microstructure, namely flat lamellar scales in opaque butterflies, in $(15,46)$ or hairs in the wing of the housefly Musca domestica (47); theoretical modelling on one type of microstructure in (48)), or even a major role of nanostructures in the overall variation $(15,47)$. Yet, these two analyses only examine one type of microstructure, thereby potentially underestimating the importance of microstructures when more types of microstructures are considered. Overlap in scales is assumed to help anisotropy in hydrophobicity (49). Here, we find that it attenuates the loss of hydrophobicity with water evaporation, thereby maintaining self-cleaning ability more efficiently.

Wing mechanical resistance is crucial for flight and geometries that limit protrusion height are more resistant to breakage and less hydrophobic (50). Several of our results suggest scale height may be limited: (i) erect piliform scales are likely bending over the membrane as shown by modelling. (ii) When piliform scales are alone, they have similar height, be they flat or erect, maybe because they bend easily, which may limit their sensitivity to breakage. (iii) Erect lamellar scales are shortened compared to flat lamellar scales, which likely increases their resistance to breakage. (iv) Erect transparent lamellar scales are densely packed, as shown in Gomez et al. (30), which can also increase their mechanical resistance. Further experiments are needed to elucidate these aspects, and clarify the role of nanostructures, as not only their presence, but their topography and its randomness have been recently suggested to play a role in determining antiwetting properties (51).

\section{Trade-off between hydrophobicity and optical transparency}

299 Using spectrometric measurements of wing direct transmittance, we found a negative relationship between contact angle and mean transmittance over 300-700 nm (Table S5, Figure 7). A 10\% increase in transmittance resulted in a $4^{\circ}$ loss in CA. While this relationship was marginally significant without controlling for phylogenetic relatedness, whatever the level of analysis (all CA values, mean CA values per individual, mean CA values per species), it was statistically important (i. e., the $95 \%$ credibility interval did not contain 0 ) in phylogeny-controlled analyses when considering all measurements or mean individual values, but less important at species level ( $90 \%$ credibility interval), likely because of weaker statistical power. In agreement with our prediction that microstructures play a major role in hydrophobicity, we find a negative relationship between hydrophobicity and transparency, a condition associated with major modifications in scale shape and density. This trade-off can be seen from the literature: the nymphalid butterfly Greta oto has been shown to exhibit a high transparency resulting from poorly dense erect piliform scales and efficient antireflective nanostructures $(35,37)$ but a weak hydrophobicity (27). Likewise, the trade-off can be seen in the dragonfly Gynacantha dravida (which has micro and nanospikes), in which distal wing parts show higher hydrophobicity but lower transmittance 
compared to proximal wing parts (52). Finding this trade-off is fully compatible with the fact that some species with nude membrane show high hydrophobicity: membrane nanostructures are at full play in species with nude membrane and can potentially efficiently reduce both reflection and water adhesion, like in the cicada Aleeta curvicosta (53). In species with erect scales, our models show a major role of microstructures and a negligible role of nanostructures in hydrophobicity. If selection for hydrophobicity is relaxed on membrane nanostructures in species with erect scales, this may explain why nanostructures are so diverse in their architecture (type, density), as recently shown in clearwing Lepidoptera (36).

\section{Hydrophobicity and latitude}

323 Compared to their temperate counterparts, species living in the tropics had a higher hydrophobicity in their transparent zone - loss of $10^{\circ} \mathrm{CA}$ for $10^{\circ}$ increase in latitude - but a similar hydrophobicity in their opaque zone (Table S6, Figure 8) All species showed superhydrophobic opaque patches (intercept above $150^{\circ}$ in Table S6, Figure 8B) and there was no relationship between the proportion of wing area occupied by transparency and latitude that could have explained the observed variations in CA (model with proportion of transparency as dependent variable, latitude effect $=0.08 \pm 0.22, t=-0.31, p=0.76$, the best model was the null model). This result is consistent with the prediction that in tropical climates where species face more humid conditions, and where rainfall can happen daily, there is a stronger selective pressure for increased hydrophobicity. While the opaque zone allows maximizing hydrophobicity in all environmental conditions, the differential in environmental conditions reveals the costs of transparency. To our knowledge, this is the first evidence for a higher hydrophobicity in more humid conditions. Scarce relevant studies have explored the link between habitat humidity and species hydrophobicity: at local geographical scale, all four cicada species studied by (6) show superhydrophobicity regardless of whether they live in dry or more humid habitats, but annual species are more hydrophobic than the species that emerges in large swarms every 17 years. Likewise, Goodwyn et al. (29) suggest that in transparent butterflies hydrophobicity may depend on lifespan and migration ability. Further studies are needed to elucidate the links between hydrophobicity and species ecology. acts on multiple features of scales (shape, orientation, coloration, density) in relation to climatic conditions. Considering more natural geometric complexity in experimental and theoretical studies on hydrophobicity should open new venues for applied physics and answer open questions like the role of randomness in nanostructures, shown to improve optical transparency (35) but suggested to impair hydrophobicity (54, but see 51$)$. 


\section{Species selection}

355

Scale type and scale insertion have been suggested to influence hydrophobicity(29); scale coloration, often involving melanin deposition which increases cuticle hardening in insects (45), could increase scale stiffness and ability to repel water droplets. Hence, we selected a set of species varying in structural strategies - scale type ( $\mathrm{N}=$ nude membrane, $\mathrm{P}=$ piliform bifid or monofid scales, $\mathrm{L}=$ shape different than piliform, hereafter called lamellar, or $\mathrm{PL}=$ association of piliform and lamellar scales), insertion ( $E=$ =erect or $F=f l a t)$, and colouration ( $C=$ coloured or $T=$ transparent) - from the study of 123 species of clearwing Lepidoptera (30). We minimized the phylogenetic relatedness between species harbouring the same type of structural strategies to increase the power of comparative analyses. We selected a total of 23 species from 10 families (Figure 1 \& 2, list in Table S7), comprising 3 species for the structural strategies (N, PFC, PEC, LFC, LFT, LEC, LET), 2 species for PLEC and LEC, and 1 species for PLET, as for some species only a limited number of specimens were present in the collections. For each species, we selected three specimens in good condition either from Paris MNHN collections or from our own private collections. 54/69 specimens (all species but Eutresis hypereia) had labels with exact collect location that could be tracked down to GPS coordinates.

\section{Hydrophobicity measurements}

For each specimen, we used a purpose-built water-droplet dispenser (a graduated pipette on a holder) and a Keyence VHX-5000 microscope (equipped with Z20 zoom) to image water droplets on butterfly wings. As a general procedure, we dropped a series of three $1 \mu \mathrm{l}$ water droplets (volume usually taken to assess hydrophobicity $(3,29))$ at three locations of the transparent and opaque zones of the dorsal side of a wing. After the water droplet was dropped (time T1), we allowed its volume to be approximately divided by two (time T2) and by four (time T3) compared to its original volume. Since evaporation kinetics depended on droplet shape, time intervals elapsed between consecutive photos were not identical from one species to another. At each time, we took a photo (Figure 1) in which we measured the static contact angle (Figure S1). Contact angle measurements were first measured on both wings and found highly repeatable (see detailed methods and results in SI, Table S8). We thus kept the same protocol, but we measured only the forewing.

\section{Measurements of wing macro, micro and nanostructure}

To characterize wing macrostructure, we took photos of the three specimens of each species using a camera (D800E Nikon, 60mm lens, annular light). We analysed photos using ImageJ (55). Given the role of wing length (4), maybe wing shape (4), and ratio of total wing area to body mass (53) on hydrophobicity and self-cleaning ability, we computed wing length, length-to-width LW ratio and the ratio of total wing area to body volume, taking the volume as a proxy for mass for dry specimens, and assuming the body to be a cylinder, for which we measured length (thorax+abdomen) and width. Using the 'rptR' package (56), we found that all wing macrostructural measurements were repeatable, i.e. that a specimen was representative of its species for all wing macrostructural variables (Table S8). 
To characterize wing microstructure (i.e. scale characteristics, presence, type, insertion, coloration, density), we imaged the dorsal side of forewing transparent and opaque zones using microscopes (Zeiss Stereo Discovery V20 and Keyence VHX-5000). We did that in one specimen per species because scale dimensions and density had already been found repeatable at zone by species level in Gomez et al. (30). Using ImageJ or the Keyence built-in tool, we measured scale density (per $\mathrm{mm}^{2}$ ), length and width $(\mu \mathrm{m})$, scale surface (in $\mu \mathrm{m}^{2}$ ) as the product of length by width, and scale coverage as the product of scale surface (expressed in $\mathrm{mm}^{2}$ ) by scale density. We counted the number of different scale types: $0=$ nude membrane, $1=$ lamellar scales or piliform scales, $2=$ combination of piliform scales and lamellar scales. For flat lamellar scales, we also computed the density of scale top layer and computed the number of layers as the ratio between density and top layer density.

We quantified scale nanostructures - width and spacing (in $\mu \mathrm{m}$ ) of longitudinal ridges present on all scales - on the detailed top-view microscopical images. We computed the ridge-ratio as ridge width to spacing (SI, Fig S2). Since museum specimens are patrimonial material, we could not damage or destroy specimens to image membrane nanostructures or scale ridge height.

\section{Optical measurements}

For one specimen per species, we measured specular transmittance from 300 to $700 \mathrm{~nm}$ as in Gomez et al. (30), using a deuterium-halogen lamp (Avalight DHS), direct optic fibres (FC-UV200-2-1.5 x 100) and a spectrometer (Avaspec-2048 L, Avantes). Wing samples were placed perpendicular at equal distance between fibres aligned $5 \mathrm{~mm}$ apart $(1 \mathrm{~mm}$ diameter spot). We took five measurements of the forewing in various points of the transparent zone. Using Avicol v6 (57), we computed the mean transmittance over [300-700] nm, which described the level of optical transparency. Optical measurements had been found highly repeatable at species level in Gomez et al. (30).

\section{Comparative analyses}

All analyses were conducted using the R environment_(58)._We conducted mixed models not controlling for phylogeny using the 'nlme' R package (59). We selected the best mixed model using AICc minimization. We used the formulated model for Bayesian mixed models with MCMC analyses controlling for phylogeny, using the 'mulTree' R package (60). Bayesian analyses used the maximum clade credibility (MCC) phylogeny obtained in Gomez et al. (30) and pruned to targeted species. Comparing classic and Bayesian mixed models allowed us to assess the influence, if any, of phylogeny on the observed relationships. For Bayesian analyses, we used uninformative priors, an Inverse-Gamma distribution (shape $=0.001$, scale $=0.001$ ) for both random effect and residual variances $(61), 2$ chains of 500,000 iterations, burn-in of 10,000 , and thinning interval of 300 . Fixed effects were considered statistically important when associated with $95 \%$ credibility intervals excluding zero, and less important (marginally important) when associated with $90 \%$ credibility interval excluding zero.

Considering all contact angle measurements, we analysed the variation in contact angle with (i) wing macrostructure descriptors - time, zone, forewing size, surface, LW ratio, the ratio of total wing area divided by body volume, and relevant two and three-way interactions -; (ii) with wing microstructure descriptors - time, zone, wing length (to correct for variation in scale dimensions), scale length, width, 
density, scale type, number of different types, scale insertion, scale colouration, number of layers, ridgeratio, and biologically relevant two and three-way interactions. (iii) To characterize spatial geometries, we analysed scale length against scale insertion for structural strategies with one scale type. For structural strategies based on both scale types (piliform and lamellar), we analyzed length ratio, density ratio and spatial association between the two scale types in relation to scale insertion (see SI for details). (iv) We analyzed the difference between contact angles predicted by various Cassie-Baxter and Wenzel models (see below) and measured contact angles at T1 to select the best models. (v) We tested for a potential trade-off between optical transparency and wing hydrophobicity, considering all measurements of contact angle, individual mean values, or species mean values at T1. (vi) Finally, we tested whether tropical species were more hydrophobic than temperate species. To do so, we related for each specimen its average $C A$ value to its latitude to the equator, the proportion of wing area occupied by transparency and wing length, while taking species as random effect, for the opaque and transparent zone separately. We also tested whether variation in the proportion of transparency could be explained by the latitude to the equator.

\section{Hydrophobicity modelling}

To assess to what extent scale micro- and nanostructure could explain the observed variation in hydrophobicity, we elaborated Cassie-Baxter model (62), whereby water only wets the top of the surface and the apparent contact angle observed at equilibrium is $\theta^{*}$.

$$
\cos \theta^{*}=\Phi_{S} \cos \theta_{S}+\Phi_{A} \cos \theta_{A}
$$

where $\Phi_{S}$ is the fraction of the droplet contact area where water is in contact with the solid, and $\Phi_{A}$ is the fraction of droplet contact area in contact with air, with $\Phi_{S}+\Phi_{A}=1 . \theta_{A}=180^{\circ}$ (contact angle of water with air) and the angle $\theta_{S}$ (also called Young angle) is the contact angle of water on an ideal smooth surface of the same material (wing membrane), set to $95^{\circ}$, as in Fang et al (15), a plausible value given the presence of epicuticular wax on the wing membrane (63), even in clearwing Lepidoptera (37). This equation is valid for water droplet size largely exceeding surface roughness wavelength (64), which is the case for $1 \mu$ l water droplets. Considering flat-topped geometries simplifies Eq1 to:

$$
\cos \theta^{*}=\Phi_{S} \cos \theta_{S}-\left(1-\Phi_{S}\right) \text {. }
$$

While $\Phi_{S}=1$ for a nude membrane, $\Phi_{S}<1$ for all the other microstructures (Figure S5 for calculations). We explored several hypotheses: piliform scales fully erect or bending over a fraction $p p=3 / 5$ of their length (the value $3 / 5$ comes from P/L length ratio $=2.6$ found for PLE strategies in Figure S4), an organization of flat lamellar scales in one layer if in low density, or several layers (NL) when in high density, models with microstructures only, or with micro and nanostructures. In the latter case, we considered water droplets contacted scale nanostructures whether flat scales (PF or LF) covered the wing membrane entirely or not, or only when scales were in high coverage, building a homogeneous horizontal surface.

We also elaborated a Wenzel model (1), whereby water fills all the pores of the surface and the wetting contact angle is primarily determined by surface roughness $r$. The apparent contact angle $\theta^{*}$ is obtained by:

$$
\cos \theta^{*}=r \cos \theta_{S}
$$


485

486

487

488

489

490

491

492

493

494

495

496

where $r$ is the ratio between the total solid surface and the projected surface, and the angle $\theta_{S}$ is the same as previously. We only included microstructures in the model since we had no measurement of scale ridge height or membrane nanostructures. While $r=1$ for a nude membrane, $r$ exceeds 1 for all the other microstructures (see Figure S7 for calculations).

\section{Acknowledgments}

This work was funded by Clearwing ANR project (ANR-16-CE02-0012), HFSP project on transparency (RGP0014/2016) and a France-Berkeley fund grant (FBF \#2015-58). We warmly thank Jacques Pierre and Rodolphe Rougerie for help with species choice, identification, and data on species ecology, Edgar Attivissimo for contributing to Keyence imaging, and Thibaud Decaëns, Daniel Herbin, and Claude Tautel for species selection and identification.

\section{References}

1. R. N. Wenzel, Resistance of solid surfaces to wetting by water. Ind. Eng. Chem. 28, 988-994 (1936).

2. W. Barthlott, C. Neinhuis, Purity of the sacred lotus, or escape from contamination in biological surfaces. Planta 202, 1-8 (1997).

3. J. Hasan, et al., Spatial Variations and Temporal Metastability of the Self-Cleaning and Superhydrophobic Properties of Damselfly Wings. Langmuir 28, 17404-17409 (2012).

4. D. Byun, et al., Wetting Characteristics of Insect Wing Surfaces. J. Bionic Eng. 6, 63-70 (2009).

5. M. Sun, G. S. Watson, Y. Zheng, J. A. Watson, A. Liang, Wetting properties on nanostructured surfaces of cicada wings. J Exp Biol 212, 3148-3155 (2009).

6. J. Oh, et al., Exploring the Role of Habitat on the Wettability of Cicada Wings. ACS Appl. Mater. Interfaces 9, 27173-27184 (2017).

7. Y. Su, et al., Nano to Micro Structural Hierarchy Is Crucial for Stable Superhydrophobic and Water-Repellent Surfaces. Langmuir 26, 4984-4989 (2010).

8. M. S. Bell, A. Shahraz, K. A. Fichthorn, A. Borhan, Effects of Hierarchical Surface Roughness on Droplet Contact Angle. Langmuir 31, 6752-6762 (2015).

9. W. Barthlott, M. Mail, C. Neinhuis, Superhydrophobic hierarchically structured surfaces in biology: evolution, structural principles and biomimetic applications. Philos. Trans. R. Soc. AMath. Phys. Eng. Sci. 374, 20160191 (2016). 
10. N. A. Patankar, Mimicking the lotus effect: Influence of double roughness structures and slender pillars. Langmuir 20, 8209-8213 (2004).

11. F. Porcheron, P. A. Monson, Mean-Field Theory of Liquid Droplets on Roughened Solid Surfaces: Application to Superhydrophobicity. Langmuir 22, 1595-1601 (2006).

12. W. Ding, M. Fernandino, C. A. Dorao, Conical micro-structures as a route for achieving superrepellency in surfaces with intrinsic hydrophobic properties. Appl. Phys. Lett. 115, 053703 (2019).

13. C. E. Cansoy, H. Y. Erbil, O. Akar, T. Akin, Effect of pattern size and geometry on the use of Cassie-Baxter equation for superhydrophobic surfaces. Colloids and Surfaces A: Physicochemical and Engineering Aspects 386, 116-124 (2011).

14. P. Tsai, R. G. H. Lammertink, M. Wessling, D. Lohse, Evaporation-Triggered Wetting Transition for Water Droplets upon Hydrophobic Microstructures. Phys. Rev. Lett. 104, 116102 (2010).

15. Y. Fang, G. Sun, Y. H. Bi, H. Zhi, Multiple-dimensional micro/nano structural models for hydrophobicity of butterfly wing surfaces and coupling mechanism. Sci. Bull. 60, 256-263 (2015).

16. A. Sanchez-Monge, J. Rodriguez Arrieta, M. Jimenez-Chavarria, A. Retana-Salazar, Observations on the Ultrastructure and Hydrophobicity of the Wings of Thirteen Neotropical Families of Diptera (insecta) with Comments on Their Flight. Acta Microsc. 24, 111-117 (2015).

17. I. N. Miaoulis, B. D. Heilman, Butterfly thin films serve as solar collectors. Ann. Entomol. Soc. Am. 91, 122-127 (1998).

18. I. C. Cuthill, et al., Disruptive coloration and background pattern matching. Nature 434, 72-74 (2005).

19. M. Stevens, C. L. Stubbins, C. J. Hardman, The anti-predator function of "eyespots" on camouflaged and conspicuous prey. Behav. Ecol. Sociobiol. 62, 1787-1793 (2008).

20. D. J. Kemp, Female butterflies prefer males bearing bright iridescent ornamentation. Proc Royal Soc B 274, 1043-1047 (2007).

21. S. Berthier, Thermoregulation and spectral selectivity of the tropical butterfly Prepona meander: a remarkable example of temperature auto-regulation. Appl Phys A-Mater 80, 13971400 (2005).

22. A. Krishna, et al., Infrared optical and thermal properties of microstructures in butterfly wings. Proc Natl Acad Sci USA 117, 1566-1572 (2020).

23. C.-C. Tsai, et al., Physical and behavioral adaptations to prevent overheating of the living wings of butterflies. Nat Commun 11, 551 (2020).

24. W. Nachtigall, Aerodynamische Messungen am Tragfluegelsystem segeinder Schmetterlinge. Journal of Comparative Physiology A 54, 210-231 (1967). 
25. N. Slegers, et al., Beneficial aerodynamic effect of wing scales on the climbing flight of butterflies. Bioinspir. Biomim. 12, 016013 (2017).

26. T. Wagner, C. Neinhuis, W. Barthlott, Wettability and contaminability of insect wings as a function of their surface sculptures. Acta Zoologica 77, 213-225 (1996).

27. N. D. Wanasekara, V. B. Chalivendra, Role of surface roughness on wettability and coefficient of restitution in butterfly wings. Soft Matter 7, 373-379 (2011).

28. Y. Fang, et al., "Micro-morphological Models for the Special Wettability of Locust and Moth Wing" in Proceedings of the 2017 5th International Conference on Mechatronics, Materials, Chemistry and Computer Engineering (Icmmcce 2017), M. Wang, X. Zhou, Eds. (Atlantis Press, 2017), pp. 330-333.

29. P. Perez Goodwyn, Y. Maezono, N. Hosoda, K. Fujisaki, Waterproof and translucent wings at the same time: problems and solutions in butterflies. Naturwissenschaften 96, 781-787 (2009).

30. D. Gomez, et al., Transparency in butterflies and moths: structural diversity, optical properties and ecological relevance. Ecological Monographs (2021) https:/doi.org/10.1101/2020.05.14.093450 (May 19, 2020).

31. M. McClure, et al., Why has transparency evolved in aposematic butterflies? Insights from the largest radiation of aposematic butterflies, the Ithomiini. Proc Royal Soc B 286, 20182769 (2019).

32. M. Arias, et al., Transparency reduces predator detection in chemically protected clearwing butterflies. Funct Ecol (2019) https:/doi.org/10.1111/1365-2435.13315.

33. M. Arias, M. Elias, C. Andraud, S. Berthier, D. Gomez, Transparency improves concealment in cryptically coloured moths. J Evol Biol 33, 247-252 (2020).

34. A. Yoshida, M. Motoyama, A. Kosaku, K. Miyamoto, Antireflective nanoprotuberance array in the transparent wing of a hawkmoth, Cephonodes hylas. Zoological Science 14, 737-741 (1997).

35. R. H. Siddique, G. Gomard, H. Holscher, The role of random nanostructures for the omnidirectional anti-reflection properties of the glasswing butterfly. Nature Communications 6 (2015).

36. C. Pinna, et al., Convergence in light transmission properties of transparent wing areas in clearwing mimetic butterflies. bioRxiv, 2020.06.30.180612 (2020).

37. A. F. Pomerantz, et al., Developmental, cellular, and biochemical basis of transparency in the glasswing butterfly Greta oto. Journal of Experimental Biology accepted, eb237917 (2021).

38. Y. M. Zheng, X. F. Gao, L. Jiang, Directional adhesion of superhydrophobic butterfly wings. Soft Matter 3, 178-182 (2007).

39. G. McHale, S. Aqil, N. J. Shirtcliffe, M. I. Newton, H. Y. Erbil, Analysis of Droplet Evaporation on a Superhydrophobic Surface. Langmuir 21, 11053-11060 (2005). 
40. M. Reyssat, J. M. Yeomans, D. Quéré, Impalement of fakir drops. EPL 81, 26006 (2007).

41. D. Quéré, Wetting and Roughness. Annual Review of Materials Research 38, 71-99 (2008).

42. X. Gao, L. Jiang, Water-repellent legs of water striders: Biophysics. Nature 432, 36-36 (2004).

43. A. Otten, S. Herminghaus, How Plants Keep Dry: A Physicist's Point of View. Langmuir 20, 24052408 (2004).

44. G. S. Watson, B. W. Cribb, J. A. Watson, Contrasting Micro/Nano Architecture on Termite Wings: Two Divergent Strategies for Optimising Success of Colonisation Flights. PLOS ONE 6, e24368 (2011).

45. M. Sugumaran, Complexities of cuticular pigmentation in insects. Pigment Cell \& Melanoma Research 22, 523-525 (2009).

46. S. N. Aideo, D. Mohanta, Unusually diverse surface-wettability features found in the wings of butterflies across Lepidoptera order and evaluation of generic and vertical gibbosity-based models. Phys. Scr. 96, 085004 (2021).

47. Q. Wan, et al., Combination of active behaviors and passive structures contributes to the cleanliness of housefly wing surfaces: A new insight for the design of cleaning materials. Colloid Surf. B-Biointerfaces 180, 473-480 (2019).

48. S. H. Sajadinia, F. Sharif, Thermodynamic analysis of the wetting behavior of dual scale patterned hydrophobic surfaces. Journal of Colloid and Interface Science 344, 575-583 (2010).

49. D. Bixler, B. Bhushan, Rice- and butterfly-wing effect inspired self-cleaning and low drag micro/nanopatterned surfaces in water, oil, and air flow. Nanoscale 6, 76-96 (2014).

50. E. Bittoun, A. Marmur, The Role of Multiscale Roughness in the Lotus Effect: Is It Essential for Super-Hydrophobicity? Langmuir 28, 13933-13942 (2012).

51. Y. Li, et al., Biomimetic Random Arrays of Nanopillars and Nanocones with Robust Antiwetting Characteristics. J. Phys. Chem. C 124, 17095-17102 (2020).

52. S. N. Aideo, D. Mohanta, Limiting hydrophobic behavior and reflectance response of dragonfly and damselfly wings. Applied Surface Science 387, 609-616 (2016).

53. G. S. Watson, S. Myhra, B. W. Cribb, J. A. Watson, Putative functions and functional efficiency of ordered cuticular nanoarrays on insect wings. Biophys. J. 94, 3352-3360 (2008).

54. M. Sun, et al., Influence of Cuticle Nanostructuring on the Wetting Behaviour/States on Cicada Wings. PLOS ONE 7, e35056 (2012).

55. C. A. Schneider, W. S. Rasband, K. W. Eliceiri, NIH Image to ImageJ: 25 years of image analysis. Nat. Methods 9, 671-675 (2012).

56. M. A. Stoffel, S. Nakagawa, H. Schielzeth, rptR: repeatability estimation and variance decomposition by generalized linear mixed-effects models. Methods Ecol Evol 8, 1639-1644 (2017). 
57. D. Gomez, AVICOL v6. a program to analyse spectrometric data. Free program available from the author upon request at dodogomez@yahoo.fr or by download from http://sites.google.com/site/avicolprogram/(2011).

58. R Development Core Team, $R$ : a language and environment for statistical computing ( $R$ Foundation for Statistical Computing, 2013).

59. J. Pinheiro, D. Bates, D. DebRoy, D. R. Sarkar, nlme: linear and nonlinear mixed effects models. R package version 3.1-145 (2020).

60. T. Guillerme, K. Healy, mulTree: performs MCMCgImm on multiple phylogenetic trees. $R$ package version 1.3.6. (2019).

61. J. D. Hadfield, MCMC methods for multi-response generalized linear mixed models: The MCMCgImm R Package. J Stat Soft 33 (2010).

62. A. B. D. Cassie, S. Baxter, Wettability of porous surfaces. Transactions of the Faraday Society 40, 546 (1944).

63. E. P. Ivanova, et al., Molecular Organization of the Nanoscale Surface Structures of the Dragonfly Hemianax papuensis Wing Epicuticle. PLOS ONE 8, e67893 (2013).

64. A. J. B. Milne, A. Amirfazli, The Cassie equation: How it is meant to be used. Advances in Colloid and Interface Science 170, 48-55 (2012). 
bioRxiv preprint doi: $\mathrm{https}$ //doi.org/10.1101/2021.10.11.463886; this version posted October 13, 2021. The copyright holder for this preprint (which was not certified by peer review) is the author/funder, who has granted bioRxiv a license to display the preprint in perpetuity. It is made available under aCC-BY-NC-ND 4.0 International license.
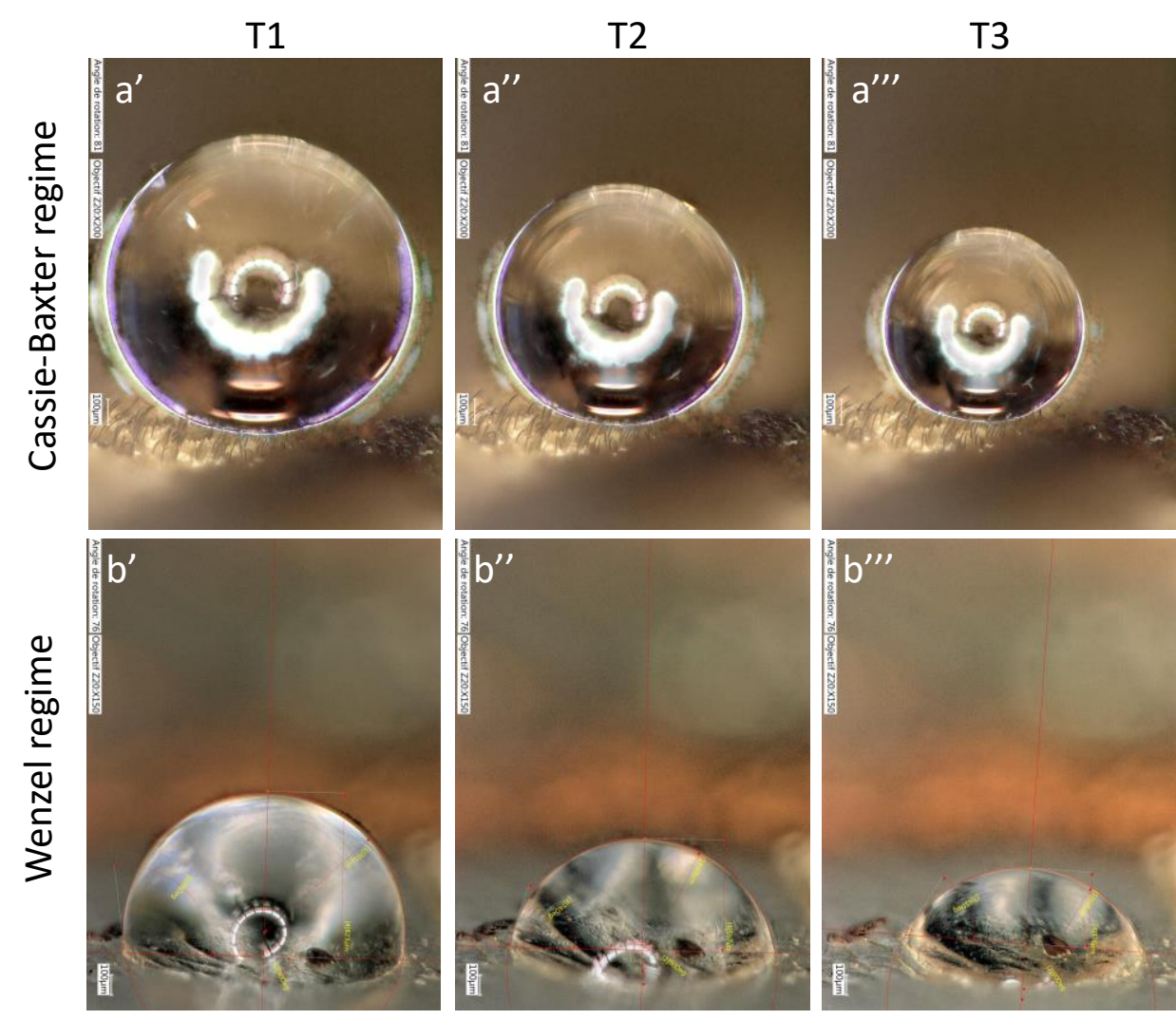

Figure 1. Examples of water droplets dropped in the transparent zone: Cassie-Baxter regime (series a) for Eutresis hypereia combining erected coloured piliform and lamellar scales and Wenzel regime (series b) for Neorcarnegia basirei with a nude membrane. Water droplet evolution is shown at different times: T1 $\left(a^{\prime}, b^{\prime}\right)$, T2 $\left(a^{\prime \prime}, b^{\prime \prime}\right)$, and at T3 $\left(a^{\prime \prime \prime}, b^{\prime \prime \prime}\right)$. 


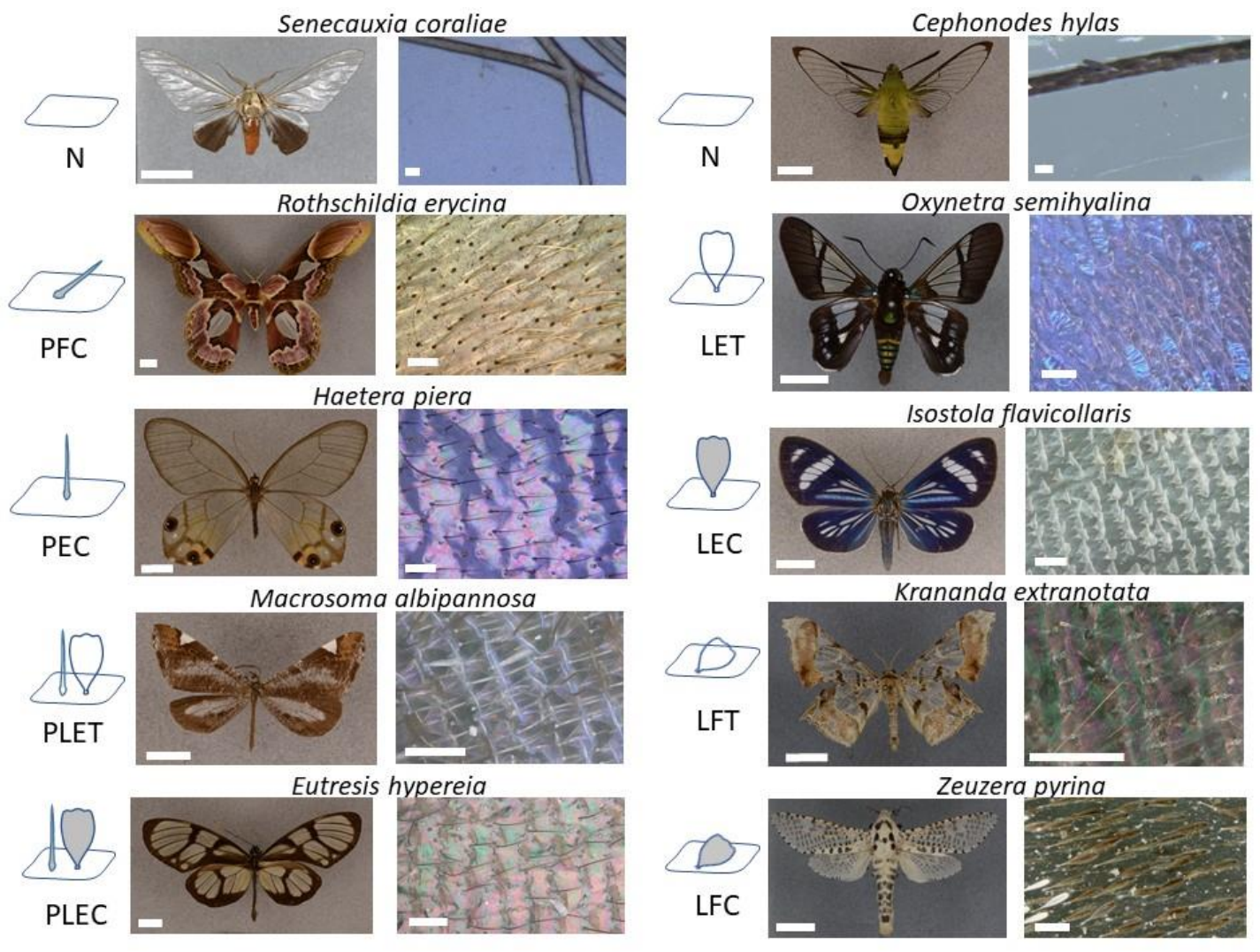

Figure 2. Examples of structural strategies in clearwing butterflies. Structural strategy is a combination of scale type (N: no scales, P: piliform scales, L: lamellar scales, PL: combination of piliform scales and lamellar scales), scale insertion ( $\mathrm{E}$ : erected, and $\mathrm{F}$ : flat), and scale colour (C: coloured, and $\mathrm{T}$ : transparent). Notice that Macrosoma albipannosa has transparent lamellar scales but coloured piliform scales. 


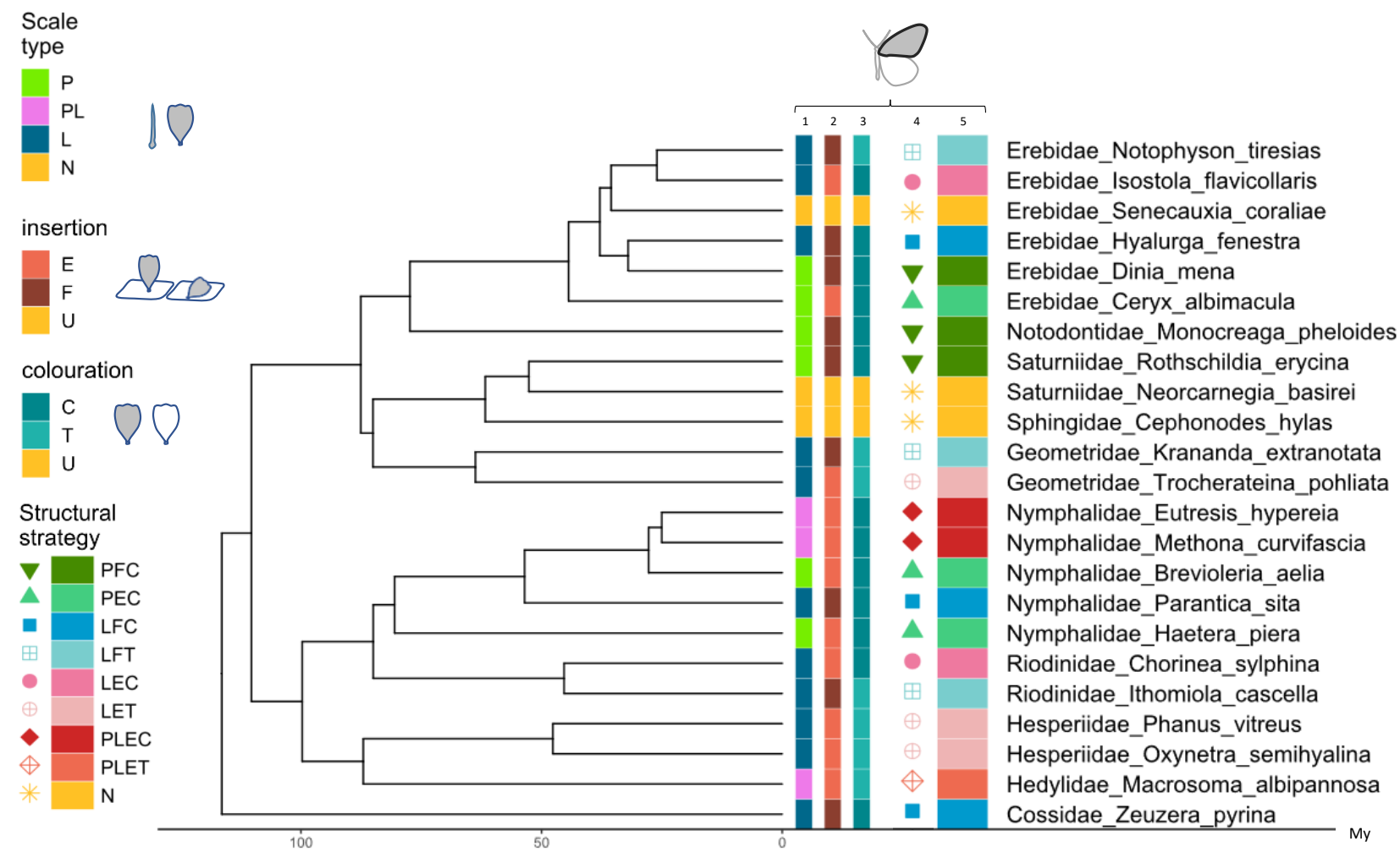

Figure 3. Phylogeny and distribution of trait values in the study species, for the forewing. Scale type (column 1), scale insertion on the membrane (column 2), scale colouration (column 3), and structural strategy (columns 4 and 5). For scale type: $\mathrm{N}=$ no scales, $\mathrm{P}=$ piliform scales, L= lamellar scales $\mathrm{PL}=$ combination of piliform scales and lamellar scales. For scale insertion on the membrane: $\mathrm{E}=$ erected, $\mathrm{F}=$ flat, $\mathrm{U}=$ undefined (for absent scales). For scale colouration: $\mathrm{C}=$ coloured, $\mathrm{T}=$ transparent, $\mathrm{U}=$ undefined (for absent scales). The strategy NUU was simplified into $\mathrm{N}$.

661 
A

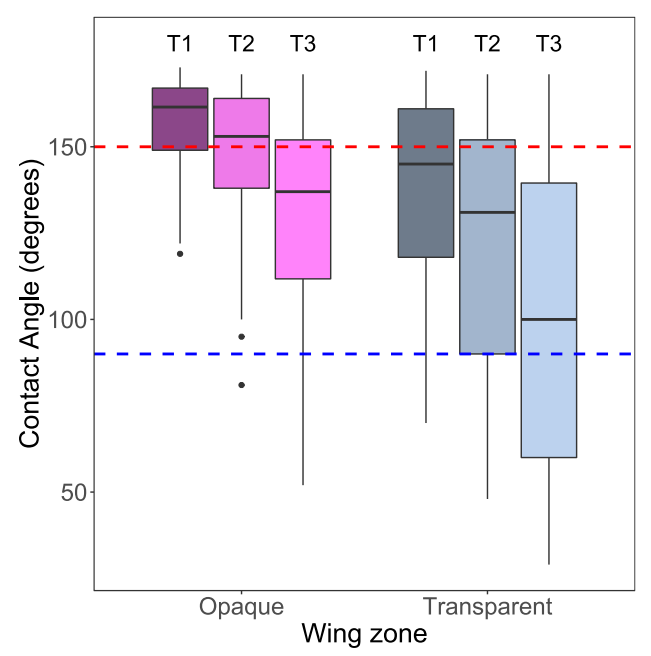

$\mathrm{C}$

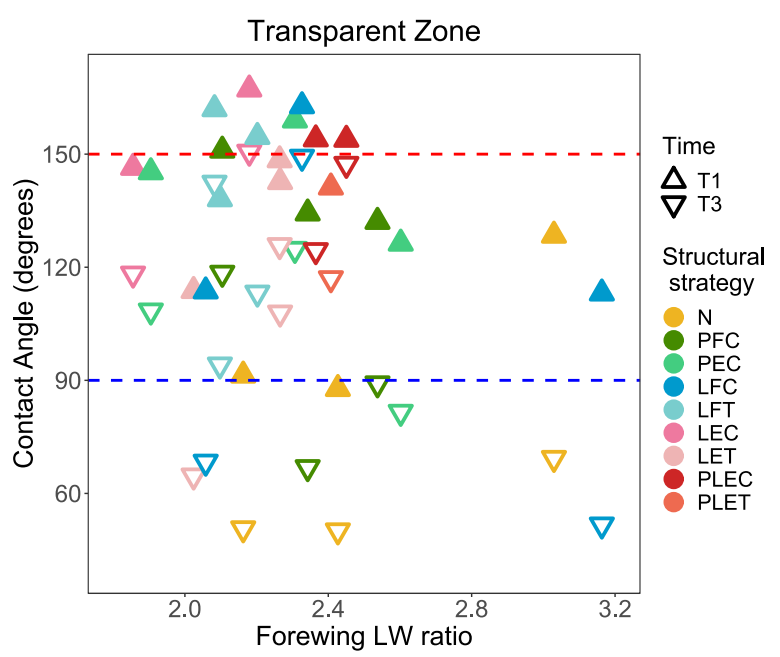

B

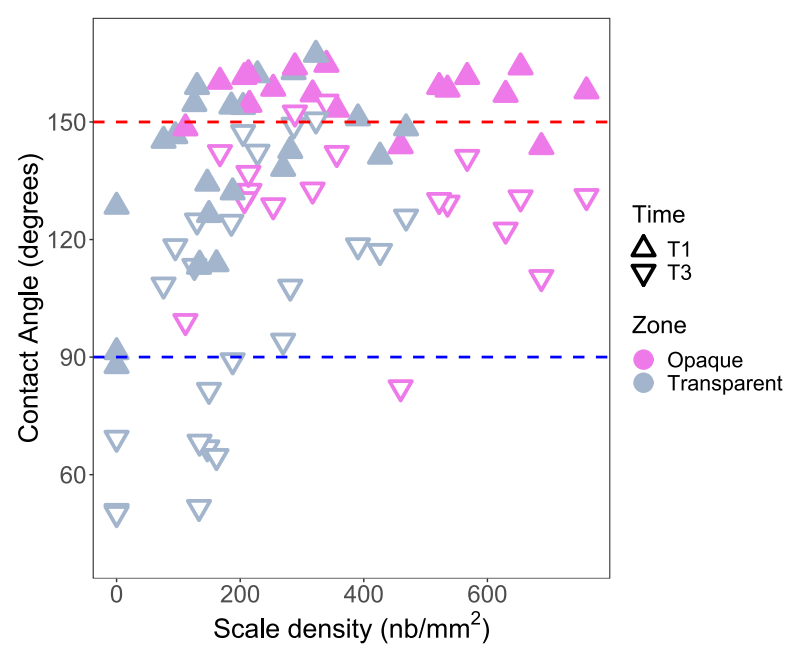

D

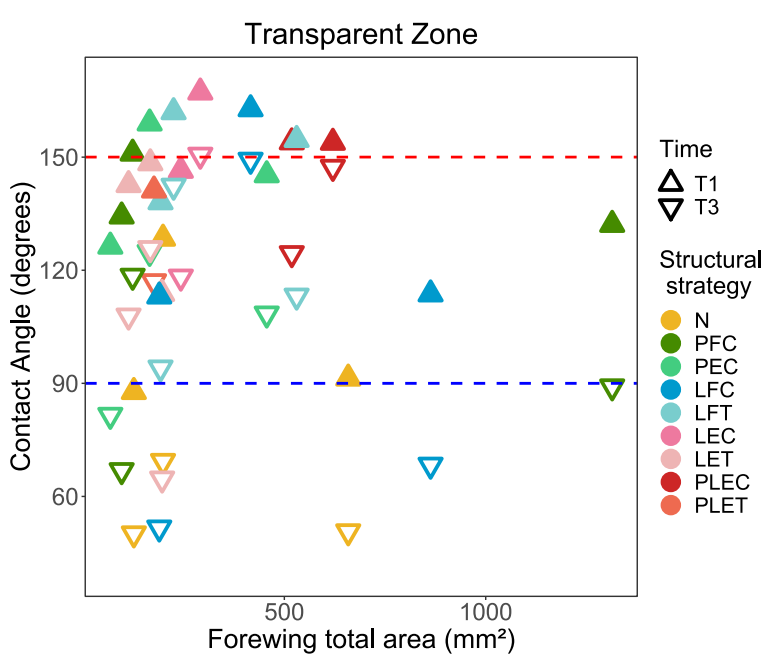

61.

670 Figure 4. Variation in contact angle with evaporation time(A) and with scale density (B) in the 671 transparent and opaque zones. Variation in contact angle with forewing length-to-width ratio (C) and total area $(D)$ in the transparent zone. Structural strategy is a combination of scale type (N: no scales, P: piliform scales, L: lamellar scales, PL: combination of piliform scales and lamellar scales), scale insertion (E: erected, and F: flat), and scale colour (C: coloured, and T: transparent). Superhydrophobic: $>150^{\circ}$ (above the red line), hydrophobic: $<150^{\circ}$ and $>90^{\circ}$; hydrophilic: $<90^{\circ}$ (below the blue line). (A) all measurements considered, $(B C D)$ mean $C A$ values for each species, zone, and time. Results are presented in Tables S1 to S3d. 


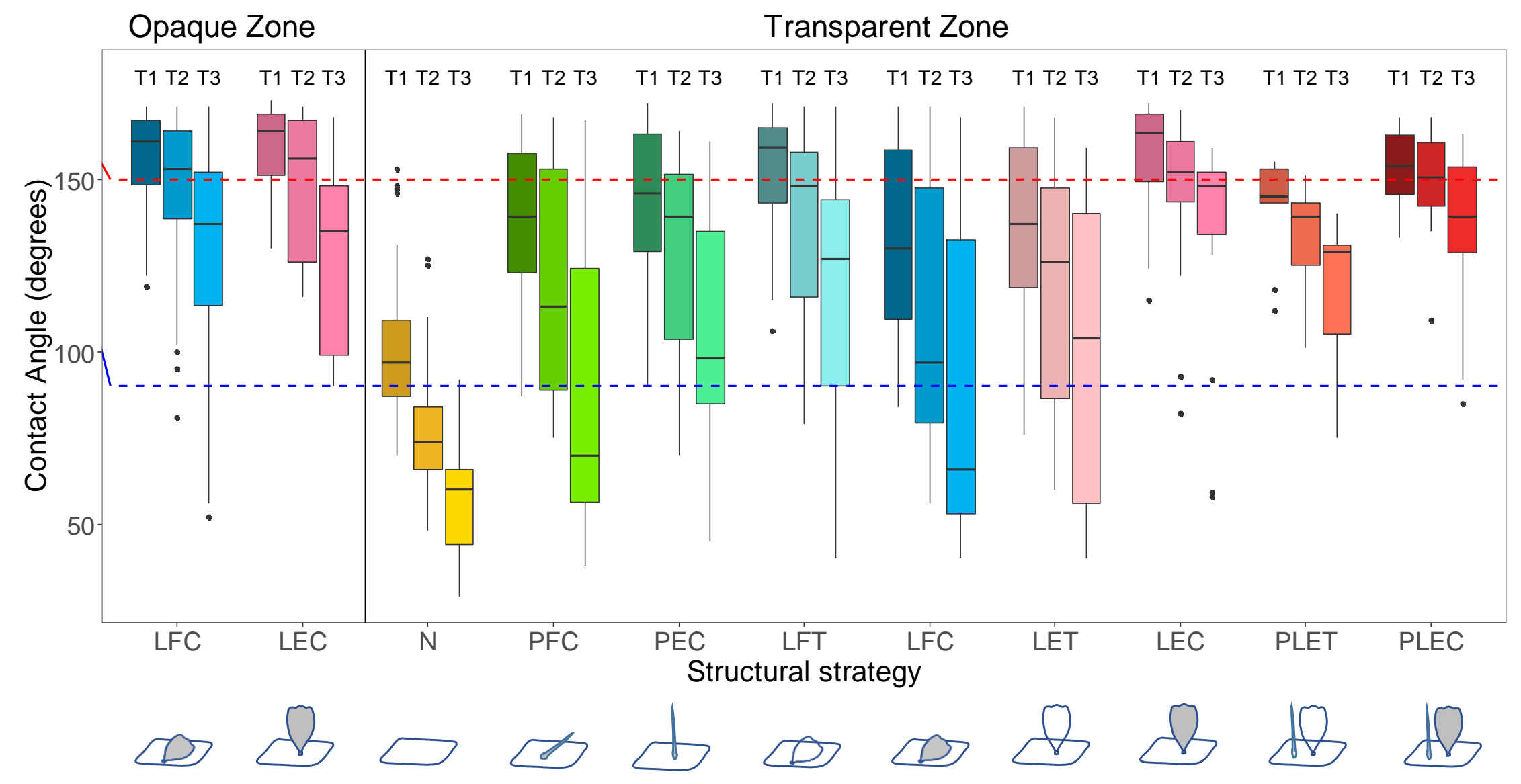

Figure 5. Variations of contact angle with wing zone, microstructure and time, i.e. water droplet size. Structural strategy is a combination of scale type (N: no scales, P: piliform scales, L: lamellar scales, PL: combination of piliform scales and lamellar scales), scale insertion (E: erected, and F: flat), and scale colour (C: coloured, and T: transparent). Superhydrophobic: $>150^{\circ}$ (above the red line), hydrophobic: $<150^{\circ}$ and $>90^{\circ}$; hydrophilic: $\angle 90^{\circ}$ (below the blue line). All individuals and droplets were considered. Results are presented in Tables S2 to S3d. 
A

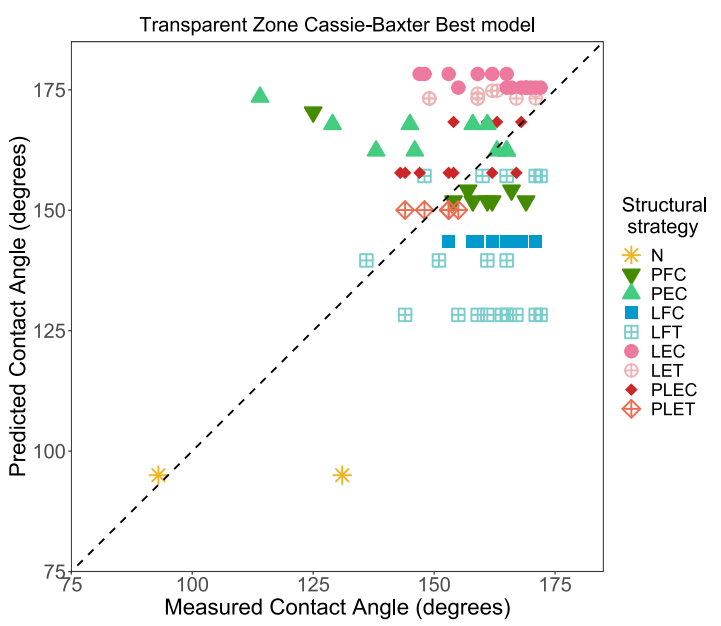

B

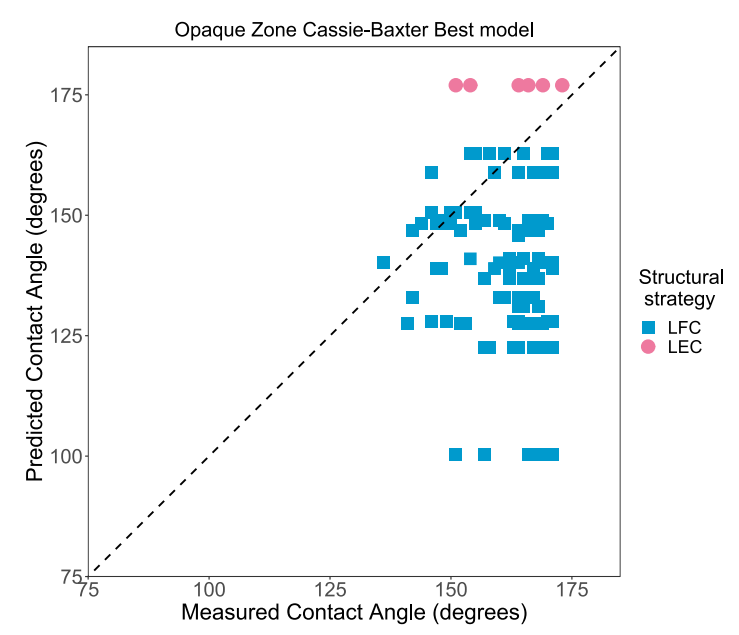

1

3 Figure 6. Comparison between contact angle values predicted by the best Cassie-Baxter model 4 for the transparent zone (A) and the opaque zone (B). For the measured values, we included all 5 the values from water droplets characterized as following a Cassie-Baxter regime, i.e. showing a $6 \mathrm{CA}>120^{\circ}$ at T3. The best model considered the effect of microstructures for all structural strategies 7 (with bending P scales when erect) and an effect of nanostructures, but only for flat lamellar scales 8 in high coverage. Structural strategy is a combination of scale type (N: no scales, P: piliform scales, 9 L: lamellar scales, PL: combination of piliform scales and lamellar scales), scale insertion (E: erect, 10 and F: flat), and scale colour (C: coloured, and T: transparent). The dashed black line represents 11 12 13 perfect agreement between prediction and measurement. We considered all the measured water droplets, and for time T1. 


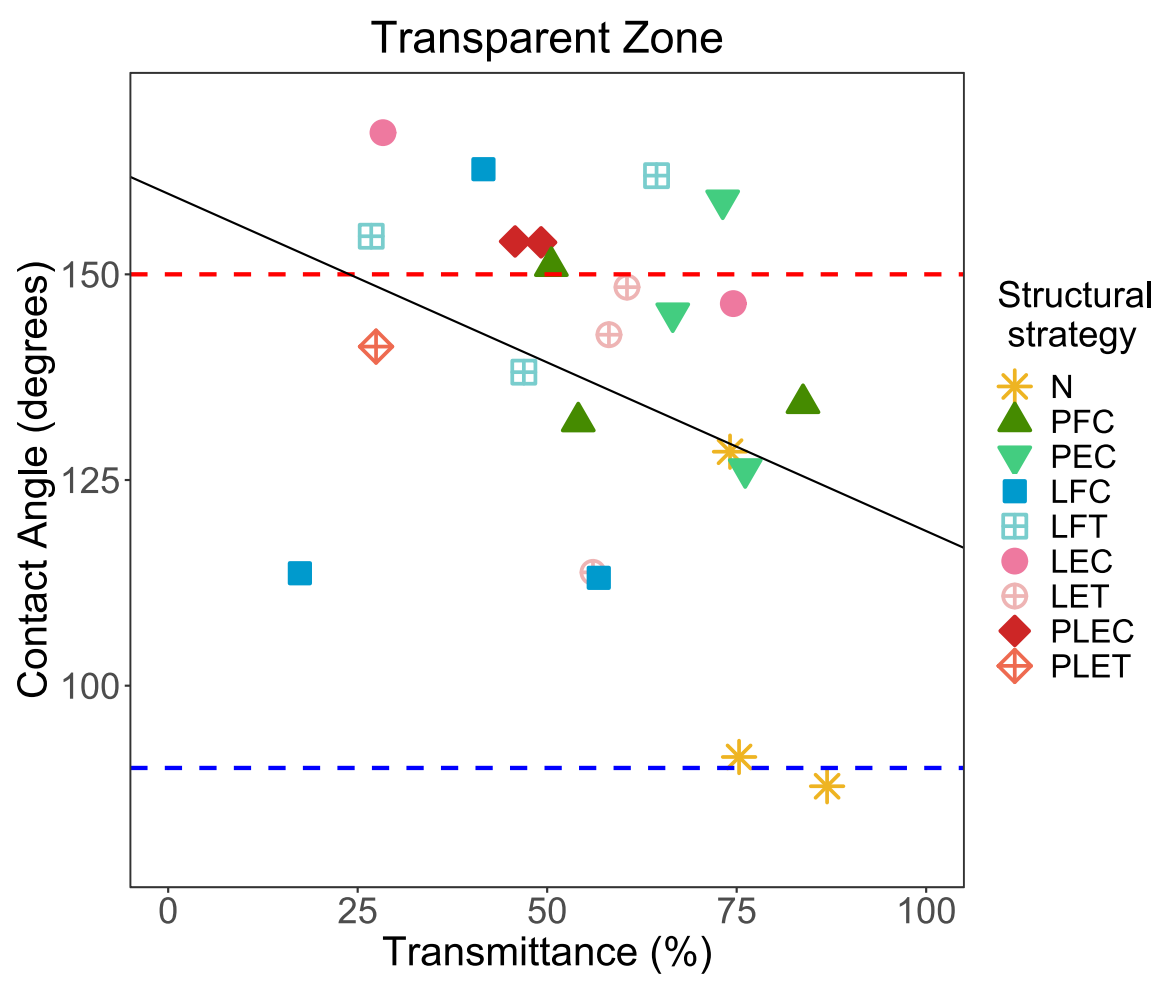

16 Figure 7. Variations of contact angle with wing transmittance for the different structural strategies. Structural strategy is a combination of scale type (N: no scales, P: piliform scales, L: lamellar scales, PL: combination of piliform scales and lamellar scales), scale insertion (E: erected, and F: flat), and scale colour (C: coloured, and T: transparent). Superhydrophobic: $>150^{\circ}$ (above the red line), hydrophobic: $<150^{\circ}$ and $>90^{\circ}$; hydrophilic: $<90^{\circ}$ (below the blue line). We considered only the mean of CA for each species, for time T1, and for the transparent zone. The black plain line indicates the significant fitted regression line based on the Bayesian model. Results are presented in Table S5. 
A

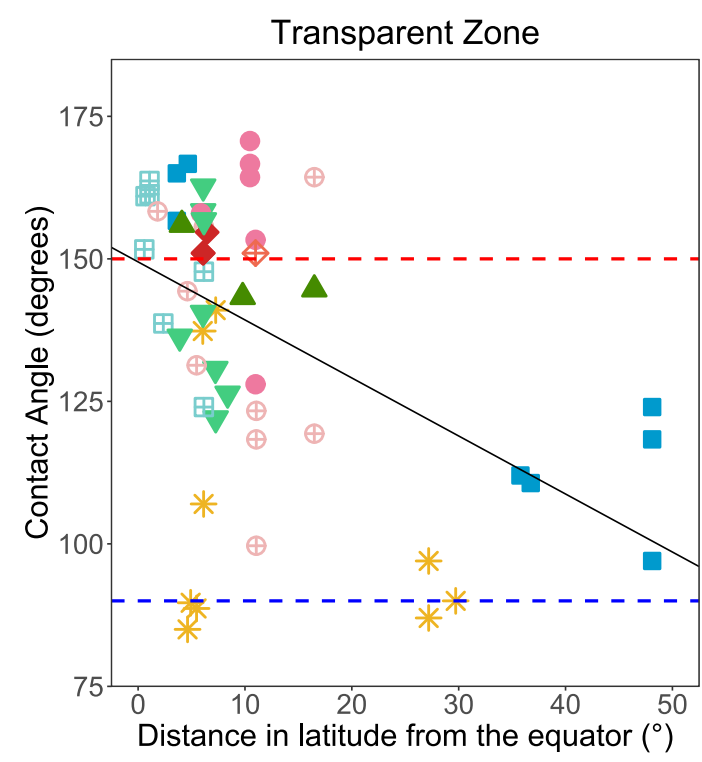

B

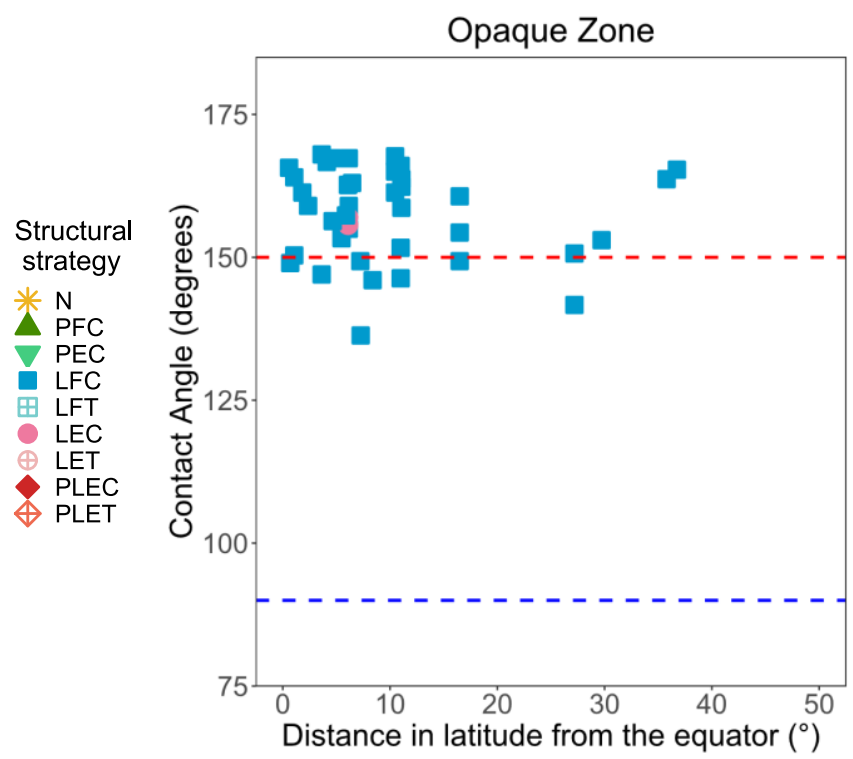

28 Figure 8. Relationship between contact angle in the transparent (A) and in the opaque (B) zone and the distance in latitude to the equator. Structural strategy is a combination of scale type ( $\mathrm{N}$ : no scales, P: piliform scales, L: lamellar scales, PL: combination of piliform scales and lamellar scales), scale insertion (E: erected, and F: flat), and scale colour (C: coloured, and T: transparent). Superhydrophobic: $>150^{\circ}$ (above the red line), hydrophobic: $<150^{\circ}$ and $>90^{\circ}$; hydrophilic: $<90^{\circ}$ (below the blue line). The black plain line in $A$ indicates the significant fitted regression line based on the Bayesian model. Results are presented in Table S6. 